\title{
Limitations of dual $x$-ray absorptiometry in children with chronic kidney disease
}

\author{
Lutz T. Weber • Otto Mehls
}

Received: 24 March 2009/Revised: 18 May 2009/Accepted: 18 May 2009/Published online: 15 July 2009

(C) IPNA 2009

\begin{abstract}
Dual x-ray absorptiometry (DXA) is the most widely used densitometric method for diagnosing osteoporosis in adults. It has also been widely adopted as a diagnostic tool in the pediatric population. The most significant limitation of DXA is its reliance on areal rather than volumetric bone mineral density (BMD), which results in an artificial underestimation of bone density in short people. Poor longitudinal growth, however, is an eminent problem in children with chronic kidney disease (CKD). There is also no evidence in children that areal BMD is predictive of future fracture risk, which is the traditional rationale for measuring $\mathrm{BMD}$ in children with $\mathrm{CKD}$. Therefore, the Kidney Disease Outcomes Quality Initiative guidelines and the current position of the International Society for Clinical Densitometry (ISCD) on pediatric patients, both of which are presented in this issue of Pediatric Nephrology, do not recommend the use of DXA in children with CKD. To date, there is no consensus on the best method to assess the degree of renal osteodystrophy in this patient population, and further collaborative efforts to correlate densitometric findings with clinical outcomes are warranted.
\end{abstract}

\footnotetext{
L. T. Weber $(\bowtie)$

Pediatric Nephrology, Dr. von Haunersches Kinderspital, University Children's Hospital, Ludwig-Maximilian's University, Munich, Germany

e-mail: Lutz.Weber@med.uni-muenchen.de

O. Mehls

Division of Pediatric Nephrology,

University Hospital for Pediatric and Adolescent Medicine, Im Neuenheimer Feld 153,

69120 Heidelberg, Germany
}

Keywords Bone mineral density

Children with chronic kidney disease · Fracture risk .

Peripheral quantitative computed tomography.

X-ray absorptiometry

\section{Introduction}

Renal osteodystrophy develops early in the course of chronic kidney disease (CKD) and reflects a multifactoral disorder that has significant prospective impact on the patient's condition. Although there is neither an etiological nor clinical reason to equate renal osteodystrophy with osteoporosis, the same technical tools used to measure osteoporosis in adult patients are also used in the pediatric population to assess bone mass and bone density. The traditional rationale for measuring bone mineral density (BMD) in children with CKD is to assess immediate and delayed fracture risk. However, to date, there is no significant published body of evidence that demonstrates the association of any parameter of bone status with fracture risk in children with CKD. In addition to fracture risk, however, we have to consider further important complications of bone disease in CKD, such as slipped epiphyses, skeletal deformities, back pain and scoliosis.

Mary B. Leonard has recently published a comprehensive review in Pediatric Nephrology on the assessment of bone status in children and adolescents with CKD [1]. One quintessence of her review is that dual $\mathrm{x}$-ray absorptiometry (DXA) cannot be recommended in children with CKD because of its significant limitations as a diagnostic tool in this patient population. This conclusion is in line with the Kidney Disease Outcomes Quality Initiative (K/DOQI) guidelines for treating bone metabolism and disease, which 
state that DXA should not be used to monitor BMD in children with CKD [2].

DXA certainly is the most widely used densitometric method for diagnosing osteoporosis in adults. Clinicians treating pediatric patients have also widely adopted this method due to its low cost, easy use, short scanning times and relatively low radiation. The main reasons why it is not recommended for children with $\mathrm{CKD}$ are:

1) The most significant limitation of DXA is its reliance on areal rather than volumetric BMD; consequently, it does not provide information on the depth of the bone. As wider and longer bones also tend to be thicker, larger bones have artificially inflated areal BMD measurements, resulting in an artificial underestimation of bone density in short people, irrespective of a potentially underlying chronic disease. This confounding factor of poor growth is definitely significant in children with CKD suffering from growth retardation. It is therefore even questionable whether DXA should be used in the growing child at all.

2) DXA does not distinguish between cortical and trabecular bone. This results in the utility of DXA being limited in the setting of increased parathyroid hormone (PTH) because trabecular and cortical bone respond differently to increased PTH activity - that is, trabecular bone increases in mass and cortical bone decreases in mass [3]. The ability to distinguish between cortical and trabecular bone may also be relevant in cases of chronic steroid therapy that result in an impairment of normal acquisition of bone mass. Via multiple pathways, steroid therapy primarily results in a reduction of trabecular bone, but it may also adversely affect cortical bone [4-8].

3) DXA provides little data on measures of bone geometry and trabecular microarchitecture. However, bone circumference, periostal and endostal dimensions and trabecular microarchitectural parameters, such as trabecular thickness, number, spacing, orientation, connectivity and the ratio of plate- and rod-like structures, all contribute to bone strength [9].

4) There is no evidence in children that areal BMD is predictive of future fracture risk [10].

Renal osteodystrophy is a condition as complex as skeletal metabolism and is influenced not only by disturbed calciotropic and somatotropic hormone axes but also by inflammatory processes. The acceptance of DXA as the sole method for assessing the degree of renal osteodystrophy is too limited, and the clinician should not use DXA in routine clinical practice for this purpose. However, the poor results may not exclusively be a problem of the method itself; they may also be due to a lack of adequate study designs. For example, the incidence of pathologic fractures is generally low in children and, therefore, the number of children needed to reach the required statistical power that would enable definite assessments of the value of areal BMD would be enormous.

Despite these drawbacks, there is no consensus on the best non-invasive method to assess the degree of renal osteodystrophy, and there are currently few adequate alternatives to DXA. Peripheral quantitative computed tomography (pQCT) undoubtedly has advantages, such as the potential to differentiate between trabecular and cortical bone and the ability to provide measures of a bone's resistance to bending forces. Unfortunately, it also does not provide information on trabecular microarchitecture. Advances in software and techniques now allow highresolution peripheral quantitative computed tomography (HR-pQCT), which may have future value in assessing bone geometry and microarchitecture by overcoming one limitation of $\mathrm{pQCT}$, i.e. that the resolution is too small to capture trabecular width and cortical thickness accurately [11]. Despite these innovations, the clinician has to consider that, when performing pQCT measurements, there are still problems of standardized scan acquisition and analysis programs, including consensus on where to mark the end of the bone in young children with large growth plates and where to target the region of interest [10, 11]. Recent advances in micro-magnet resonance imaging (DMRI) allow detailed structural information to be obtained from the DMRI "virtual bone biopsy". A pilot study in young adults on maintenance hemodialysis suggests that DMRI may have the potential to characterize the structural implications of renal bone disease [12].

In conclusion there is still a need for further collaborative efforts to correlate densitometric findings with clinical outcomes in children with CKD.

The official positions of the International Society for Clinical Densitometry (ISCD) are reported in this issue of Pediatric Nephrology [13]. The Pediatric Position Development Conference organized by the ISCD again concludes that DXA is of less value in children with CKD. Although not specifically focused on CKD, we can learn from these positions on how to assess, interpret and report on bone densitometry. These guidelines are well edited according to quality of evidence, strength of recommendation, applicability and necessity.

\section{References}

1. Leonard MB (2007) A structural approach to the assessment of fracture risk in children adolescents with chronic kidney disease. Pediatr Nephrol 22:1815-1824

2. Kidney Disease Outcomes Quality Initiative (K/DOQI) (2005) Clinical practice guidelines for bone metabolism and disease in children with chronic kidney disease. Am J Kidney Dis 46:1-103 
3. Parfitt AM (1998) A structural approach to renal bone disease. J Bone Miner Res 13:1213-1220

4. Leonard MB, Bachrach LK (2001) Assessment of bone mineralization following renal transplantation in children: Limitations of DXA and confounding effects of delayed growth and development. Am J Transplant 1:193-196

5. Saland JM (2004) Osseous complications of pediatric transplantation. Pediatr Transplant 8:400-415

6. Tenbrock K, Kruppa S, Mokov E, Querfeld U, Michalk D, Schönau E (2000) Analysis of muscle strength and bone structure in children with renal disease. Pediatr Nephrol 14:669-672

7. Lettgen B, Jeken C, Reiners C (1994) Influence of steroid medication on bone mineral density in children with nephritic syndrome. Pediatr Nephrol 8:667-670

8. Rueth E, Weber LT, Schoenau E, Wunsch R, Seibel MJ, Feneberg R, Mehls O, Toenshoff B (2004) Analysis of the functional muscle-bone unit of the forearm in pediatric renal transplant recipients. Kidney Int 66:1694-1706
9. Mittra E, Rubin C, Qin YX (2005) Interrelationship of trabecular mechanical and microstructural properties in sheep trabecular bone. J Biomech 38:1229-1237

10. Specker BL, Schoenau E (2005) Quantitative bone analysis in children: Current methods and recommendations. J Pediatr 146:726-731

11. Burrows M, Liu D, McKay H (2009) High-resolution peripheral QCT imaging of bone micro-structure in adolescents. Osteoporos Int. doi:10.1007/s00198-009-0913-2

12. Wehrli FW, Leonard MB, Saha PK, Gomberg BR (2004) Quantitative high-resolution magnetic resonance imaging reveals structural implications of renal osteodystrophy on trabecular and cortical bone. J Magn Reson Imaging 20:83-89

13. Bianchi ML, Baim S, Bishop NJ, Gordon CM, Hans DB, Langman CB, Leonard MB, Kalkwarf HJ (2009) Official positions of the International Society for Clinical Densitometry (ISCD) on DXA evaluation in children and adolescents. Pediatr Nephrol. doi:10.1007/s00467-009-1249-z 